\title{
In search of a strong visual recency effect
}

\author{
DENNY C. LECOMPTE \\ Rice University, Houston, Texas
}

\begin{abstract}
When a sequence of visual stimuli is presented in a fixed location, immediate serial recall of the sequence is characterized by only a small recency effect. According to Battacchi, Pelamatti, and Umiltà (1990), the distribution of visual stimuli over space, as well as time, greatly enhances the recency effect. After an initial failure to find a strong visual recency effect with distributed presentation (Experiment 1), in the remaining experiments an attempt was made to more closely approximate Battacchi et al.'s methodology by eliminating articulatory suppression (Experiments 2-7), using their stimuli (Experiments 3-7), blocking conditions (Experiments 4-7), requiring written rather than typed responses (Experiments 5-7), and using their list length (Experiments 6 and 7). Nevertheless, even when their method was followed as closely as possible (Experiment 7), distributed presentation did not produce a strong visual recency effect. The influence of distributed presentation on the visual recency effect would seem to be, at best, limited.
\end{abstract}

A fundamental characteristic of immediate memory is its tendency to preserve many of the sensory qualities of the perceived stimulus (James, 1890). In fact, these sensory qualities often play a functional role in immediate mernory. For example, experiments have shown that in serial recall, auditory items are remembered better than visual items (see Penney, 1989, for a review). This modality effect is typically restricted to items at or near the end of a list: Relative to recall of earlier items, there is a large increase in recall for the most recently presented items of auditory lists, but only a small increase in recall for visual lists. Although there has been no lack of theoretical speculation about the modality effect (e.g., Frankish, 1989; Frick, 1988; Glenberg \& Swanson, 1986; Nairne, 1988, 1990; Penney, 1989), Crowder's (1986) characterization of existing research remains apt: "The classical auditory-visual modality effect is large and reliable, but still poorly understood" (p. 268).

The standard procedure for investigating the modality effect involves presenting auditory and visual lists one item at a time, with all items occurring at the same location. In other words, the items are distributed temporally, but not spatially. A possible explanation of the modality effect is that this lack of spatial distribution places visual lists at a disadvantage. It has been argued that the auditory system is inherently better at coding temporal information, whereas the visual system is better at coding spatial information (see, e.g., Gardiner, 1983; Glenberg \& Swan-

This research was supported by National Institute of Mental Health Grant MH35873 awarded to Michael J. Watkins. The article benefited from suggestions by Marco W. Battacchi, Robert G. Crowder, Stephanie H. LeCompte, Rober W. Proctor, Henry L. Roediger III, and Arthur Samuel. I would like to express my appreciation to Stanley B. Fish and Amy Sayle for their contributions to Experiment 1. I would especially like to thank Michael J. Watkins who advised me throughout this project. Requests for reprints should be sent to Denny $\mathbf{C}$. LeCompte, Department of Psychology, Louisiana State University, Baton Rouge, LA 70803. son, 1986; Metcalfe, Glavanov, \& Murdock, 1981). This argument is supported by the fact that the modality effect is weakened when subjects are not required to recall the items in their order of occurrence (Murdock \& Walker, 1969; Watkins, 1974). If differences in encoding affect immediate memory, changes in the spatial distribution of visual items could influence the modality effect. Indeed, a number of researchers have reported that presenting visual items simultaneously (which distributes them spatially, but not temporally) produces better overall recall than presenting them sequentially (e.g., Crowder, 1966; Mackworth, 1962). Moreover, Frick (1985) has found that memory span for visual items presented simultaneously does not differ significantly from memory span for auditory iterns presented sequentially; in fact, he found a slight numerical advantage for simultaneous presentation.

A particularly striking example of the effect of spatial distribution on the modality effect has recently been reported by Battacchi, Pelamatti, and Umiltà (1990, Experiment 1). They wanted to test the hypothesis that distributing visual items spatially would increase recall, even when the items were also distributed temporally. In their experiment, subjects serially recalled six-item lists in one of four conditions: (1) an auditory condition, (2) a fixed visual condition, (3) a horizontally distributed visual condition, and (4) a vertically distributed visual condition. The first two conditions form the standard basis for demonstrating the modality effect. Of interest were the third and fourth conditions, which were designed to test the effect of distributed presentation. In these conditions, the stimuli, permutations of the syllables $p e, p i$, and $p u$, appeared in six different spatial locations, each to the right of or below the previous one.

A large recency effect was found with auditory presentation, but only a minimal effect was found with fixed visual presentation, thereby replicating the typical modality effect. More importantly, a substantial recency effect 
occurred in each of the two distributed presentation conditions. The data for all four conditions are shown in the first panel of Figure 1. ${ }^{1}$ It is worth noting that the recency effects found with the two distributed conditions were not statistically different from each other, nor were they statistically different from the auditory recency effect; however, all three recency effects were reliably larger than the typically small effect shown in the fixed visual presentation condition. Battacchi et al. (1990) concluded that "these findings, which challenge the classic, auditoryvisual modality effect, suggest that any general model of the modality effect must take into consideration the modality-specific processing of time and space" (p. 657).

Before such broad conclusions are to be accepted, it would seem prudent to explore the boundary conditions of the finding because Battacchi et al. (1990) used only one type of stimulus, one list length, one set of instructions, and so on. ${ }^{2}$ On first reading Battacchi et al.'s report, I had reason to believe that their results might be limited in generality. The belief was based on the results of an unpublished experiment, reported here as Experiment 1 .

\section{EXPERIMENT 1}

The original purpose of this experiment concerned the advantage of simultaneous visual presentation over sequential visual presentation (see, e.g., Crowder, 1966). If this advantage occurred because the simultaneously presented stimuli were distributed in space, then sequentially presented stimuli should also be remembered better when the stimuli are distributed in space. To test this notion, subjects studied eight-letter lists, each presented for a total of $4 \mathrm{sec}$, either simultaneously or sequentially. In the sequential presentation condition, the successive letters were either distributed in space or shown in the same place. Immediately following presentation, the subjects recalled the letters in serial order. Frick (1985; Frick \& DeRose, 1986) had previously found that preventing subvocalization of visually presented list items tended to induce reliance on a visual code. The subjects in this experiment were therefore required to silently mouth the syllable "blah" during all conditions. The simultaneous condition is included in the description of the experimental methods, even though it does not bear directly on the issue of visual recency.

\section{Method}

Materials and Design. The stimuli were 45 eight-letter lists. The letters for each list were chosen randomly, without replacement, from the letters $R, L, S, Q, J, G, M, H, Y$, and $B$. They were presented in capitals on a Macintosh Plus computer screen in 20-point bold Geneva font.

The lists were divided into three 15-list blocks. For any given subject, each block was assigned to a different presentation condition. In the sequential-fixed condition, each of the letters in a list appeared alone in the center of the computer screen for $.5 \mathrm{sec}$ and was immediately followed by the next letter, with no discernible interval between them. In the sequential-distributed condition, each of the letters in a list appeared alone on the computer screen for $.5 \mathrm{sec}$, but in locations different from those of the other letters. The first letter appeared near the left-hand side of the screen, and each subsequent letter appeared approximately $1 \mathrm{~cm}$ to the right of the one that preceded it. In the simultaneous condition, all eight letters appeared on screen at the same time, filling the eight locations used in the sequential-distributed condition. The eight letters remained on the screen for $4 \mathrm{sec}$, which was the total presentation time for the other conditions, and they were erased from the screen simultaneously. The letters appeared on the same horizontal axis in all conditions.

Although all of the subjects saw the same 45 lists in the same order, the presentation condition of each block of 15 lists was rotated across subjects such that each list served in each condition equally often.

Procedure. The subjects were tested individually or in small groups; however, as was true for all of the experiments reported here, even when tested in a group, each subject worked at his or her own computer. They saw short lists of letters appear on a computer screen. The computer emitted a beep $.5 \mathrm{sec}$ before the beginning of each list. At the sound of the beep, the subjects were told to begin silently mouthing the syllable "blah" and to continue until the end of the list. The experimenter monitored the subjects to ensure compliance.

After each list, the subjects were allowed $8 \mathrm{sec}$ to recall the letters in the order of their appearance for the sequential conditions and from left to right for the simultaneous condition. They were told to leave spaces for the letters that they did not remember. All responses were written. No backtracking was allowed.

Subjects. The subjects were 21 Rice University undergraduates.

\section{Results}

The means are plotted as a function of serial position in the second panel of Figure 1. Comparisons involving the simultaneous presentation condition are omitted because they are irrelevant to the question at hand. A replication of the Battacchi et al. (1990) finding would require that the recency effect in the distributed condition be appreciably larger than the recency effect in the fixed condition. Following Battacchi et al., the recency effect was defined as the difference in probability of recall between the last and next-to-last items. It was .20 in the fixedsequential condition and .19 in the distributed-sequential condition. Thus, the distributed condition failed to show any advantage. ${ }^{3}$

\section{Discussion}

This experiment showed no evidence that distributed presentation produces a strong visual recency effect, implying that Battacchi et al.'s (1990) results are limited in generality. This implication seemed worth pursuing further. The remaining six experiments were designed to identify the variables that determine when a strong visual recency effect occurs and when it does not. Each experiment more closely approximated the method of Battacchi et al., until, in the final experiment, an attempt was made to replicate the Battacchi et al. experiment as closely as possible. To foreshadow, the critical variables could 

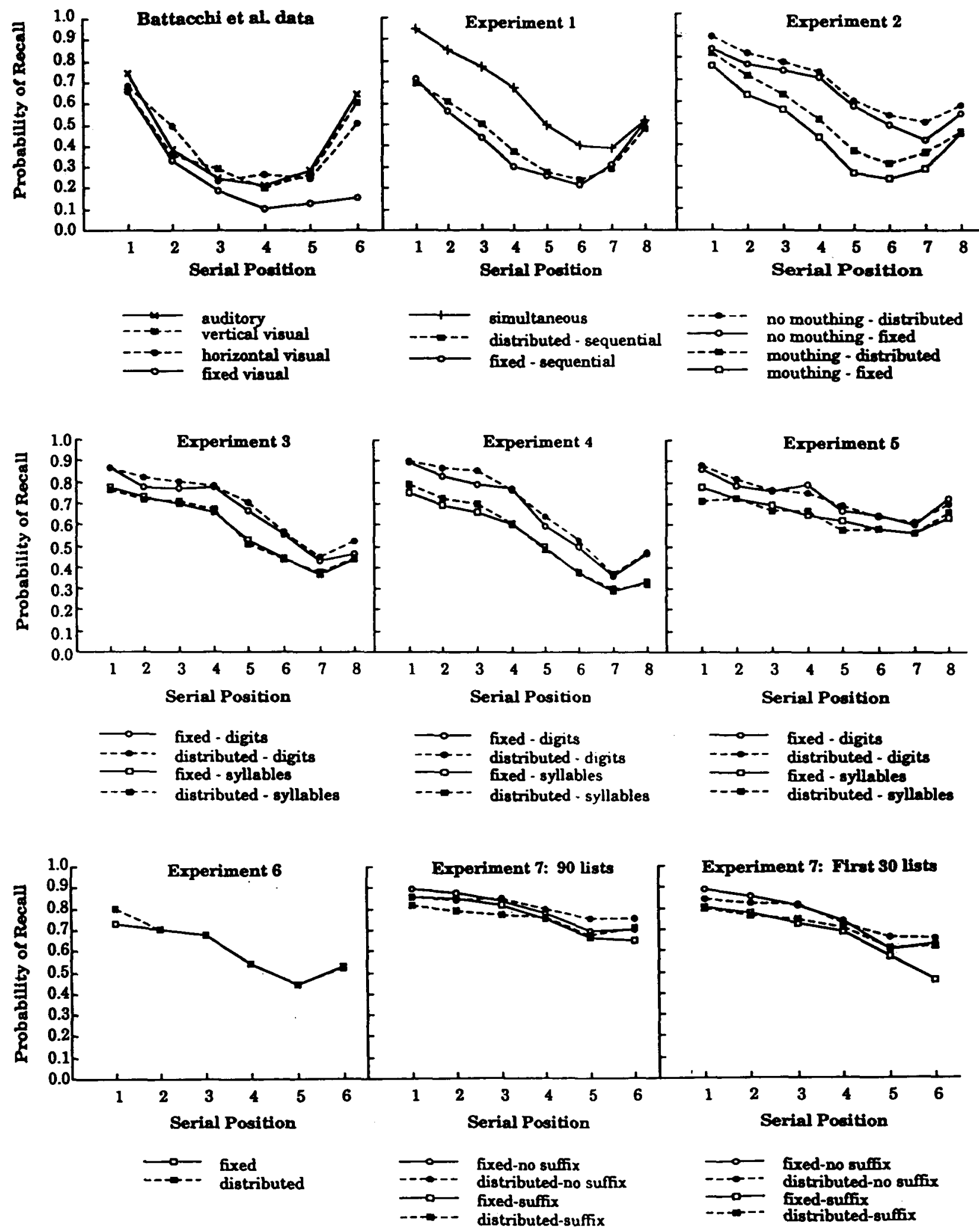

Figure 1. Probability of correct recall as a function of serial position and presentation condition for Battacchi, Pelamatti, and Umilta (1990) and for Experiments 1-7. The data for the first panel were reconstructed from the raw data of Battacchi et al. 
not be isolated because in none of the six experiments, including the direct replication, did distributed presentation give rise to a strong visual recency effect.

\section{EXPERIMENT 2}

Unlike the subjects in Battacchi et al.'s (1990) experiment, the subjects in Experiment 1 were required to mouth an irrelevant item throughout list presentation. It might have been expected that mouthing would have magnified any visual recency effects because articulatory suppression has been shown to increase subjects' reliance on visual short-term memory (Frick, 1985; Frick \& DeRose, 1986). Nevertheless, for reasons that are not entirely clear, it seems to have inhibited recency. If so, distributed presentation would be expected to produce a strong recency effect only when no mouthing was required. This hypothesis was tested in Experiment 2.

\section{Method}

Materials and Design. The stimuli were 100 lists, each comprising the digits 1-8 in random order. The lists were assigned to the fixed and distributed presentation conditions randomly, with the constraint that both the first 50 and the second 50 lists include 25 lists of each kind. A complementary version of the 100-list series was created, in which every fixed list became a distributed list and every distributed list became a fixed list. Each version of the series was presented to half of the subjects. Each of these two groups of subjects was divided into two subgroups, in one of which the subjects silently mouthed "blah" during the first 50 lists, and in one of which they silently mouthed "blah" during the second 50 lists. Thus, there were four experimental conditions (fixed lists with mouthing, fixed lists without mouthing, distributed lists with mouthing, and distributed lists without mouthing), with the order in which these conditions occurred being counterbalanced across subjects. In addition to the 100 lists already described, there were four practice lists.

All stimuli were presented in 24-point Geneva font on a Macintosh Plus computer. In the fixed presentation condition, the digits appeared in a single location at the center of the screen. In the distributed condition, each digit appeared in one of eight locations. The distributed locations were on the same horizontal axis as was the fixed location, but they were distributed evenly across the computer screen, with approximately $2 \mathrm{~cm}$ between adjacent locations.

Procedure. The subjects were tested in small groups. They saw lists presented at a rate of two digits per second. Each digit replaced the previous one with no discernible interval between them. For some lists, the digits appeared in the same spatial location; for others, they appeared in eight different locations in left-to-right order.

The subjects were required to mouth "blah" during either the first or the second half of the lists. Mouthing was required only during list presentation. For the lists in which mouthing was not required, the subjects were asked not to move their lips or in any way vocalize the digits. The experimenter monitored subjects' compliance during the presentation of all lists.

Immediately following the end of each list, the subjects tried to recall the digits in serial order by typing them on the computer keyboard. As each digit was typed, it appeared in the lower half of a rectangle shown in the center of the screen. There were eight evenly spaced dots in the upper half of the rectangle, and each digit entered in the lower half was aligned with a dot in the upper half. The dots were included to help the subjects position recalled digits. The subjects entered an asterisk in place of any item that they could not recall, and they were not allowed to backtrack. After making eight responses, the subjects hit the return key, which caused the computer screen to clear and, after $1 \mathrm{sec}$, the next list to begin.

Before beginning the experiment, the subjects were given four practice lists, one in each of the four conditions. The order of conditions was the same for all of the subjects.

Subjects. The subjects were 28 Rice University undergraduates.

\section{Results}

The means for each condition are shown in the third panel of Figure 1. If the discrepancy between the results of Experiment 1 and the results of Battacchi et al. (1990) were due to the mouthing requirement in Experiment 1, distributed presentation should have enhanced the recency effect in the no-mouthing condition, but not in the mouthing condition. It was consistent with the results of Experiment 1 that when mouthing was required, there was no advantage in the size of the recency effect for the distributed condition (.09) in comparison with the fixed condition (.16). Contrary to expectations, however, when mouthing was not required, the recency effect in the distributed condition (.08) still failed to show any advantage over that in the fixed condition (.12) ${ }^{4}$

Although the mouthing requirement was not the reason for the lack of a strong visual recency effect in either this experiment or Experiment 1, for the sake of consistency with Battacchi et al.'s procedure, this requirement was dropped from the remaining experiments.

\section{EXPERIMENT 3}

Another potentially important difference between the present method and that of Battacchi et al. (1990) is that they used the syllables $p e, p i$, and $p u$ as stimuli, whereas I used letters in Experiment 1 and digits in Experiment 2. In Experiment 3, these discrepancies were eliminated by using Battacchi et al.'s stimuli. Also, the timing of the stimuli was brought into line with that of Battacchi et al. by including a brief interval between successive list items. To determine whether any evidence of a strong recency effect was due to the stimuli or their temporal schedule, digits were also included as stimuli.

\section{Method}

Materials and Design. The stimuli were 160 eight-item lists. Half of the lists were constructed by sampling randomly, without replacement, from the digits 1-9. The other half of the lists were constructed by sampling randomly from the syllables $p e, p i$, and $p u$, with the constraint that no syllable could occur more than three times in a list. The same lists were presented to all of the subjects; however, for each subject, $\mathbf{4 0}$ of the digit lists and $\mathbf{4 0}$ of the syllable lists were assigned to the fixed condition, and the other 40 lists of each kind were assigned to the distributed presentation condition. Moreover, the order of occurrence of all 160 lists was randomized separately for each subject. The items appeared in the same font and in the same screen locations as in Experiment 2.

Procedure. Each list item appeared on the computer screen for $200 \mathrm{msec}$ and was followed by a 300-msec interval before the next one appeared. This was the same timing as that used by Battacchi et al. (1990). Also, as in Battachi et al.'s experiment, the subjects 
were asked not to speak, whisper, or in any way vocalize the items as they were presented. The experimenter monitored their compliance.

At the end of each list, the subjects recalled by typing their answers on the computer keyboard. For the digit lists, the subjects responded on the number keypad. They hit the " 0 " key, which had been re-labelled "BLANK," to indicate a recall failure. A small square represented each "blank" response. For the syllable lists, the subjects responded by pressing the " $=$," the "I," and the "*" keys on the number keypad, which had been labelled "PE," "PI," and "PU." The place markers were eight evenly spaced pairs of dots. Each keypress caused the corresponding syllable or blank symbol to appear on the computer screen aligned under the appropriate pair of dots. After eight responses, the screen cleared, and, $1 \mathrm{sec}$ later, presentation of the next list began.

After every block of 40 lists, a message appeared on the screen, instructing the subjects that they could take a short break. Most of the subjects paused for only a few seconds.

Subjects. The subjects were 16 Rice University undergraduates.

\section{Results}

The results are summarized in the fourth panel of Figure 1 . The most important fact to note about these data is that the recency effects were of the small magnitude typically found with visual presentation; in none of the four conditions was a large recency effect evident. Nevertheless, the effect of distributed presentation was analyzed statistically. For digit lists, the recency effect for distributed presentation $(.08)$ showed an advantage over the effect for fixed presentation (.03), but this advantage was only marginally reliable $[t(15)=1.49, p=.08]$. When syllables served as stimuli, the recency effect for distributed presentation (.07) showed no advantage at all over the effect for fixed presentation (.08). There was no reliable interaction between presentation condition and type of stimulus $[t(15)=1.13, p=.28]$.

\section{Discussion}

Although this was the first experiment to show a numerically larger recency effect for distributed as opposed to fixed presentation, the advantage occurred only when digits were used as stimuli, and even then, the effect was quite small and only marginally reliable. Moreover, a close examination of the fourth panel of Figure 1 reveals that the recency effects in both syllable conditions and in the digits condition with distributed presentation are very similar in magnitude $(.07, .08$, and .08$)$, whereas the recency effect in the remaining condition is approximately half the size of these others (.03). This pattern of means suggests that for the digits, the recency effect was depressed in the fixed condition rather than enhanced in the distributed condition. More important than any small differences between fixed and distributed presentation is the fact that none of these effects resembled the strong visual recency found by Battacchi et al. (1990).

\section{EXPERIMENT 4}

Battacchi et al. (1990) manipulated the fixed and distributed conditions between subjects rather than within subjects. Perhaps the consistent presentation of distributed lists allowed their subjects to develop strategies that took advantage of the items' spatial distribution. One way to more closely approximate a between-subject manipulation without sacrificing the statistical power associated with a within-subject manipulation is to present all of the trials for a particular condition as a block rather than intermixed, as they were in Experiments 2 and 3. Hence, Experiment 4 was essentially the same as Experiment 3, except that the data for one presentation condition were collected before the data for the other presentation condition were collected.

\section{Method}

The 160 lists from Experiment 3 served as stimuli in this experiment as well. For half of the subjects, the first 80 lists consisted of digits, and for the other half of the subjects, the first 80 lists consisted of syllables. For half of the subjects within each of these two subject groups, presentation was fixed for the first $\mathbf{4 0}$ digit lists and first 40 syllable lists and it was distributed for the second 40 lists of each kind. For the other half of the subjects, presentation was distributed for the first $\mathbf{4 0}$ digit lists and first $\mathbf{4 0}$ syllable lists and it was fixed for the second 40 lists of each kind. Thus, the order in which each condition occurred was counterbalanced across four equal groups of subjects. In all other respects, the method was identical to that of Experiment 3. The subjects were 12 Rice University undergraduates.

\section{Results}

The data for Experiment 4 are summarized in the fifth panel of Figure 1. For the digit lists, the recency effect with distributed presentation $(.10)$ was no larger than the effect with fixed presentation (.10). Similarly, for the syllable lists, the recency effect with distributed presentation (.02) did not exceed the effect with fixed presentation (.05). Hence, despite the blocking of conditions, distributed presentation did not give rise to a large visual recency effect.

\section{EXPERIMENT 5}

The purpose of Experiment 5 was to check the possibility that my failure to replicate Battacchi et al.'s (1990) finding occurred because Battacchi et al.'s subjects gave written responses, whereas my subjects, except for those in Experiment 1, typed their responses on the computer keyboard. Although written responses alone were not sufficient to reveal an effect of distributed presentation in Experiment 1, they might do so in combination with the various changes in method introduced in Experiments 2, 3, and 4. Accordingly, Experiment 5 was identical to Experiment 4, except that the subjects wrote their responses.

\section{Method}

The method differed from that of Experiment 4 only in that the subjects recalled each list in writing on a response sheet ruled with rows of eight blanks. The subjects worked from left to right without backtracking, leaving spaces for any items they could not recall. Twelve Rice University undergraduates served as subjects.

\section{Results}

The results are summarized in the sixth panel of Figure 1. Again, there was no hint of a large visual recency 
effect with distributed presentation. For the digit lists, the magnitude of the recency effect for distributed presentation (.09) was no larger than the magnitude of the effect observed for fixed presentation (.13). For the syllable lists, the recency effect for distributed presentation (.10) was numerically larger than the effect for fixed presentation (.07), but this advantage was not statistically reliable $[t(11)=0.91, p=.19]$.

\section{EXPERIMENT 6}

Battacchi et al. (1990) found that distributed presentation increased the size of the recency effect not only when the stimuli were distributed horizontally, but also when they were distributed vertically. In all of the experiments reported thus far, horizontally distributed presentations were used. Experiment 6 tested the possibility that a strong visual recency effect might be found more readily with vertically distributed presentation. It also tested the possibility that when the list length was shortened to six syllables, as in Battacchi et al., distributed presentation would elicit a strong visual recency effect. Only nonsense syllables were used because six-digit lists were recalled too easily.

\section{Method}

Materials and Design. The stimuli were 60 six-item lists, each comprising the syllables pe, $p i$, and $p u$. Following Battacchi et al. (1990), each list comprised two presentations of each syllable in an order that was random except for the constraint that a given syllable could not follow any other syllable more than once. The lists appeared in the same order for all subjects, but for half of the subjects the first 30 lists appeared in the distributed condition and the last 30 lists appeared in the fixed condition, and for the other half of the subjects the order of conditions was reversed.

The syllables were presented in capital letters in 24-point Geneva font on a Macintosh Plus computer. In the fixed presentation condition, the syllables appeared in a single location at the center of the screen. In the distributed condition, each syllable appeared in one of six locations. The distributed locations were on the same vertical axis as the fixed location, but they were distributed evenly down the computer screen with approximately $1.25 \mathrm{~cm}$ between any two locations.

Procedure. The subjects were shown the six-syllable lists at an onset-to-onset rate of $.5 \mathrm{sec}$; however, each syllable appeared for only $200 \mathrm{msec}$. For some lists, all of the syllables appeared in the same spatial location; for others, they appeared in six different locations, in top-to-bottom order. At the end of each list, the subjects recalled the syllables on response sheets ruled with rows of six blanks. They wrote their responses from left to right without backtracking, leaving spaces for any syllables that they could not recall.

Subjects. The subjects were 16 University of Houston undergraduates.

\section{Results}

The results are summarized in the seventh panel of Figure 1 . The recency effect for distributed presentation was .08; for fixed presentation, it was .09 . Thus, there was no advantage in recency for distributed presentation.

\section{FURTHER ANALYSES OF EXPERIMENTS 4, 5, AND 6}

In Experiments 4, 5, and 6, each subject saw all of the lists of one experimental condition before seeing lists of any other condition; hence, each experiment can be treated as a between-subject design by limiting data analysis to the initial block of lists. Because of the small number of subjects in each of these experiments, the means for any condition within an experiment would be very unstable, and the statistical power of the between-group comparisons would be low. By combining similar data across experiments, however, one can limit the severity of these problems.

For each of the subjects in Experiments 4, 5, and 6 who initially received distributed presentation, a recency effect was computed that was based only on the first block of lists. Likewise, for each of the subjects in these three experiments who initially received fixed presentation, a recency effect was computed that was based only on the first block of lists. With 20 subjects in each condition, the recency effect for distributed presentation was .10, and the recency effect for fixed presentation was .11. Thus, even when these experiments are treated as between-subject designs, there is no advantage for distributed presentation.

Note that in order to maximize the number of subjects contributing to the analysis, no distinction was made between subjects who saw syllables and subjects who saw digits. Even when the distinction is made, however, no reliable advantage of distributed presentation is found for digits or for syllables.

\section{EXPERIMENT 7}

The goal of Experiments 2-6 was to replicate the strong visual recency effect found by Battacchi et al. (1990). None of these experiments succeeded. The present experiment was an attempt to find a strong recency effect by directly replicating their method.

Before reporting the replication, it is necessary to point out one detail of the Battacchi et al. (1990) experiment that was not described in the introduction. Specifically, in each of their four conditions, half of the lists were followed by a suffix item, the syllable pa. The other half of the lists were followed by a square (or a tone in the auditory condition). It is well established that auditory recency effects are decreased considerably by the introduction of a nominally irrelevant auditory item (Crowder \& Morton, 1969); furthermore, such suffix effects are not usually found for visual stimuli with fixed presentation (see, e.g., Morton \& Holloway, 1970). Battacchi et al., however, included the suffix item in some of the lists to test the possibility that a suffix effect would emerge when visual presentation was distributed in space. They did in fact find a reliable suffix effect with the two distributed presenta- 
tion conditions, but not with the fixed presentation condition. Because Battacchi et al. manipulated the suffix condition within subjects, this variable could not be left out of the present replication, even though it is not central to the question being asked.

\section{Method}

Materials and Design. The stimuli were 90 six-syllable lists. The syllables were $p e, p i$, and $p u$; each occurred twice in each list, and their ordering within each list was random, except for the constraint that one syllable could not follow the same syllable more than once. The 90 lists were divided into six blocks of 15 lists each. Two practice lists were also constructed.

A random half of the subjects were assigned to the fixed presentation condition. The other half were assigned to the distributed presentation condition. Furthermore, following Battacchi et al. (1990), half of the subjects within each of these two groups saw a suffix item (the syllable pa) following the lists in the odd-numbered blocks; the other half of the subjects within each group saw the same suffix item following the lists in the even-numbered blocks. The first 30 lists conformed precisely to the Battacchi et al. design, and the remaining 60 lists repeated that design twice more.

The syllables were presented in capital letters in 18-point Geneva font, which closely approximated the height $(5 \mathrm{~mm})$ and width (4 mm) of Battacchi et al.'s (1990) syllables. The screen locations used for presentation in this experiment were the same as in Experiments 3-5 and had been chosen to approximate the locations used by Battacchi et al. The suffix item appeared at the center of the screen for the fixed presentation group and approximately $2 \mathrm{~cm}$ to the right of where the final item had been for the distributed presentation group.

Procedure. The subjects were tested in small groups. The subjects were asked not to whisper or in any way vocalize the syllables as they were presented; the experimenter monitored their compliance. Two practice lists were given, one with a suffix and one without.

Each trial began with a tone; a slight departure from Battacchi et al.'s (1990) method was that the tone was played through headphones connected to the computer. One half second after the tone, the syllables appeared at an onset-to-onset rate of $.5 \mathrm{sec}$, with an on time of $\mathbf{2 0 0} \mathrm{msec}$. One half second after the onset of the final syllable, either the syllable $p a$ (the suffix condition) or a square approximately the same size as the syllables (the no-suffix condition) appeared for $200 \mathrm{msec}$. The subjects were told that they were not to begin recall until they saw one of these two signals and not to include these items in their response. They responded by writing the syllables on response sheets ruled with rows of six blanks. They worked from left to right with no backtracking, leaving spaces for syllables that they could not recall. They were allowed $10 \mathrm{sec}$ for the recall of each list. Note that, except where specified, the procedure followed Battacchi et al.'s in every detail provided in their report.

Subjects. The subjects were 32 Rice University undergraduates.

\section{Results and Discussion}

Battacchi et al. (1990) included only 30 lists in their experiment. Therefore, in addition to analyzing the complete results, I also analyzed the results from the use of only the first 30 lists. The eighth panel of Figure 1 summarizes the results with all 90 lists. The last panel of Figure 1 summarizes the results with only the first 30 lists.

Analysis for all 90 lists. As is apparent from even the briefest visual comparison of the first and eighth panels of Figure 1, the results here do not resemble those re- ported by Battacchi et al (1990). The critical result was that Battacchi et al.'s strong recency effect with distributed presentation was not replicated.

The recency effect found with distributed presentation was compared with that found with fixed presentation, first in the no-suffix condition, and then in the suffix condition. In the no-suffix condition, the advantage in the size of the recency effect for distributed presentation (.02) over fixed presentation (.01) was small and not reliable $[t(30)=0.56, p=.29]$. In the suffix condition, the advantage of distributed $(.03)$ over fixed $(-.01)$ presentation was marginally reliable $[t(30)=1.34, p=.09]$. Note that Battacchi et al. did not show (or expect to show) that distributed presentation increased the size of the recency effect for the suffix condition.

Analysis for the first 30 lists. The data for the first 30 lists, shown in the last panel of Figure 1, are not quite the same as the complete results, but neither do they resemble those of Battacchi et al. (1990; see the first panel of Figure 1). Most importantly, in the no-suffix condition, the recency effect for the distributed presentation condition $(-.004)$ was no larger than the recency effect for the fixed presentation condition (.02). In the suffix condition, on the other hand, the recency effect for the distributed presentation condition (.01) was reliably larger than the effect for the fixed presentation condition [ -.11 ; $t(30)=2.72, p=.005]$. As stated above, a distributed presentation advantage in the suffix condition is not relevant to the present question. In any event, this advantage for distributed presentation was not the result of a strong recency effect; rather, it was the result of a negative recency effect in the fixed presentation condition. The strong visual recency effect reported by Battacchi et al. was completely absent from these data, regardless of suffix condition.

Additional analyses. One concern with the present experiment was that recall was, on average, much higher than that observed in the Battacchi et al. (1990) experiment. The overall probability of recall in their fixed and horizontally distributed presentation conditions (no suffix) was .27 and .41 , respectively, whereas in Experiment 7 (in the first 30 lists), the overall probability of recall in both of these conditions was .75 . Note that recall in Battacchi et al.'s experiment was unusually low: With only three possible stimuli, their subjects could have been expected to show a score of .33 purely by guessing. I am unable to explain this low level of recall; however, it is possible that the effect of distributed presentation on the visual recency effect is suppressed at the relatively high levels of recall found in Experiment 7.

To check this possibility, each subject's data were rescored for the two no-suffix conditions, using only a subset of the first 30 lists. This subset was selected so that the overall recall of each subject closely approximated the overall recall reported by Battacchi et al. (1990) in the corresponding condition. As many lists as possible were used from each subject; however, the number varied between individuals. For 8 of the 16 subjects in the dis- 
tributed condition and 4 of the 16 subjects in the fixed condition, no lists could be used because their recall was too high. After rescoring, the overall probability of recall was $.27(S D=.053)$ in the fixed condition and .41 $(S D=.039)$ in the distributed condition, just as in Battacchi et al.'s experiment. Note that the rules for selecting this data were based solely on the overall level of recall and were therefore independent of the recency effect. At issue was whether a strong recency effect would emerge in the distributed condition, given that the overall level of recall had been lowered to that of Battacchi et al.'s subjects.

For the distributed presentation condition, the adjusted probability of recall for each of the six serial positions was $.57, .46, .49, .33, .24$, and .37 . For the fixed presentation condition, the adjusted probability of recall for each of the six serial positions was $.66, .35, .12, .14$, .10 , and .25 . The important point to note about this analysis is that the recency effect for the distributed presentation condition (.13) was actually smaller than the effect for the fixed presentation condition (.15). Hence, even with potential ceiling effects removed, there was no advantage for the distributed presentation condition.

\section{SUMMARY AND CONCLUSIONS}

The effect of spatially distributing visual list items was examined in a series of seven experiments. Contrary to the findings of Battacchi et al. (1990), the distribution of the successive list items in space did not give rise to a strong visual recency effect. In Experiment 1, which was conducted without knowledge of Battacchi et al.'s work, the effect of distributed visual presentation was examined with subjects' subvocal articulation suppressed: No effect of distributed presentation was found. The remaining experiments were successive approximations to Battacchi et al.'s method, each one an attempt to identify the factors controlling the effect of distributed presentation. They eliminated as critical factors the suppression of subvocal speech (Experiment 2), type of stimuli (Experiment 3), blocking of conditions (Experiment 4), method of response (Experiment 5), method of spatial distribution, and list length (Experiment 6). Finally, Experiment 7 was an attempt to directly replicate the critical part of Battacchi et al.'s experiment. As much as possible, I used their stimuli, their experimental design, and their procedure. Nevertheless, no strong recency effect was found. In fact, in the critical no-suffix condition, distributed presentation did not increase the magnitude of the visual recency effect at all. In short, in none of the experiments was there any evidence of the large visual recency effect reported by Battacchi et al.: The recency effects obtained were of the modest size typically associated with visual presentation.

It must be acknowledged that no replication can be exact. Even in Experiment 7, which was the closest to Battacchi et al.'s (1990) experiment, the subjects spoke
English rather than Italian as their primary language. Although the stimuli ( $p e, p i$, and $p u$ ) can reasonably be described as meaningless in either language, the possibility remains that the effect of the stimuli was different in the two languages. If so, this would be an interesting finding in its own right; however, it would not change the conclusion drawn here-namely, that distributed presentation does not generally lead to a strong visual recency effect.

Any failure to replicate raises the question of whether there was enough statistical power associated with the attempted replication to detect the effect of interest. To analyze the power associated with an experiment, it is necessary to determine the effect size that is sought. Effect size was defined here in terms of $d$, which is estimated as the difference between the means being compared divided by their pooled standard deviation (Cohen, 1988). In Battacchi et al.'s (1990) experiment, for the horizontally distributed condition, $d=2.21$ (for the vertically distributed condition, $d=2.63$ ). All seven experiments reported here were powerful enough to detect an effect that large (or larger) at an alpha level of .05 (one-tailed) with a probability of greater than .995 . What if the effect were present, but smaller than that reported by Battacchi et al.? If the effect were only three quarters as large $(d=$ 1.66 ), it would have been detected with a probability of .99 or greater in any of the seven experiments. In fact, even if the effect were only half as large $(d=1,11)$, it would have been detected in all but two of the experiments with a probability greater than .90 , and in the two other experiments, it would have been detected with a probability of greater than .80 . In brief, the failure to teplicate Battacchi et al. in the present series of experiments cannot reasonably be attributed to a lack of statistical power.

In addition to the evidence reported here, data from other studies are relevant to the comparison of fixed and distributed visual presentation. For example, in studying the effect of simultaneous presentation and suppression of subvocal speech on visual short-term memory, Frick (1985) compared simultaneous presentation with both fixed presentation and distributed presentation. The fixed and distributed conditions yielded memory span estimates of 4.3 and 4.1 items, respectively. Frick did not report data for individual serial positions, making it impossible to determine the extent of any recency effects; nevertheless, the failure to find any advantage for distributed presentation in terms of overall recall is contrary to the results reported by Battacchi et al.

Another relevant experiment has been reported by McDowd and Madigan (1991, Experiment 5; see also Hitch \& Morton, 1975). They presented nine-digit lists on a computer screen, either in a central, fixed location or in a $3 \times 3$ spatial array, with the location of each list item selected independently of its temporal order. They failed to find any enhancement in recency or in overall recall from distributing the digits spatially, and they speculated that Battacchi et al. (1990) enhanced visual recency 
with spatial distribution because confusable nonsense syllables were used rather than digits. Experiments 3-7 rule out that explanation.

The effect of distributed visual presentation has also been studied by Surprenant and Crowder (1991). In two unpublished experiments, they tried to find the strong visual recency effect reported by Battacchi et al. (1990). In their first experiment, each of their subjects saw ninedigit lists, half of which were distributed vertically during presentation and half of which were fixed in the center of the screen during presentation. Each digit was presented for $500 \mathrm{msec}$, with pauses of 250,500 , or $750 \mathrm{msec}$ after the third and sixth digits. Distributed presentation did not enhance the visual recency effect in any condition. The second experiment differed from the first in that the fixed and distributed conditions were varied between subjects. Distributed presentation again failed to enhance the visual recency effect. The results of these experiments therefore reinforce the conclusions drawn here.

Although the data reported here argue against the visual recency effect's being enhanced by distributed presentation, they do not argue against the existence of substantial visual recency effects in general. In fact, such effects have been reported by a number of researchers. Substantial recency effects have been found with lip-read stimuli (see, e.g., Campbell \& Dodd, 1980), with stimuli presented in American Sign Language (see, e.g., Shand \& Klima, 1981), and, at least under some conditions, with stimuli that change physically during their presentation. For example, substantial visual recency effects were found when subjects were asked to recall the directions in which a series of small boxes moved across a computer screen (Glenberg, 1990; Kallman \& Cameron, 1989). Also, visual recency effects can be greatly enhanced with the use of free recall, rather than serial recall, instructions (see, e.g., Murdock \& Walker, 1969).

Battacchi et al.'s (1990) finding of a large visual recency effect is particularly striking because the enhancement of recency was achieved without altering the subjects' task or the nominal stimulus. Moreover, the size of the enhanced visual recency effect essentially eliminated the modality effect. Their results suggested that the modality effect amounts to little more than an artifact of the standard presentation procedure. Indeed, had their results proved to be generalizable, many theoretical accounts of the modality effect and, more generally, of immediate memory would have required extensive revision. The results of the present experiments suggest that such revisions would be premature. The effect was sought, without success, under a wide variety of conditions, including conditions that approximated the original Battacchi et al. conditions as closely as possible. Although it is impossible to rule out all conditions under which distributed presentation might increase the visual recency effect, the experiments reported here indicate that such conditions are, at best, severely limited.

\section{REFERENCES}

Battacchi, M. W., Pelamatti, G. M., \& Umilta, C. (1990). Is there a modality effect? Evidence for visual recency and suffix effects. Memory \& Cognition, 18, 651-658.

CAmpbell, R. DODD, B. (1980). Hearing by eye. Quarterly Journal of Experimental Psychology, 32, 85-99.

CoHEN, 1. (1988). Statistical power analysis for the behavioral sciences (2nd ed.). Hillsdale, NI: Eribaum.

Crowder, R. G. (1966). Visual presentation of stimuli in immediate memory. Psychonomic Science, 6, 449-450.

Crowder, R. G. (1986). Auditory and temporal factors in the modality effect. Journal of Experimental Psychology: Leaming, Memory, \& Cognition, 12, 268-278.

Crowder, R. G., \& Morton, J. (1969). Precategorical acoustic storage (PAS). Perception \& Psychophysics, 5, 365-373.

Frankish, C. (1989). Perceptual organization and precategorical acoustic storage. Joumal of Experimental Psychology: Leaming, Memory, \& Cognition, 15, 469-479.

Frick, R. W. (1985). Testing visual short-term memory: Simultaneous versus sequential presentations. Memory \& Cognition, 13, 346-356.

Frick, R. W. (1988). Issues of representation and limited capacity in the auditory short-term store. British Journal of Psychology, 79, 213-240.

Frick, R. W., DeRose, A. (1986). The suffix effect and preattentive unit-fommation in visual short-term memory. Caradian Joumal of Psychology, 40, 97-108.

Gardiner, J. M. (1983). On recency and echoic memory. Philosophical Transactions of the Royal Society of London: Series $B, 312,267-282$.

GlenberG, A. M. (1990). Common processes underlie enhanced recency effects for auditory and changing-state stimuli. Memory \& Cognition, 18, 638-650.

Glenberg, A. M., \& Swanson, N. C. (1986). A temporal distinctiveness theory of recency and modality effects. Joumal of Experimental Psychology: Leaming, Memory, \& Cognition, 12, 3-15.

HITCH, G. J., Morton, J. (1975). The unimportance of explicit spatial information in serial recall of visually presented lists. Quanerly Joumal of Psychology, 27, 161-164.

JAMES, W. (1890). Principles of psychology. New York: Holt

KallmaN, H. J. . CAMERoN, P. (1989). Enhanced recency effects with changing-state and primary-linguistic stimuli. Memory \& Cognition, 17, 318-328

MACKworTh, J. F. (1962). The effect of display time upon the recall of digits. Canadian Journal of Psychology, 16, 48-54.

MCDowD, J., \& MADIGAN, S. (1991). Ineffectiveness of visual distinctiveness in enhancing immediate recall. Memory \& Cognition, 19, 371-377.

Metcalfe, J., Glavanov, D., \& Murdock, M. (1981). Spatial and temporal processing in the auditory and visual modalities. Memory \& Cognition, 9, 351-359.

Morton, J., Holloway, C. M. (1970). Absence of a cross-modal "suffix effect" in short-term memory. Quarterly Joumal of Experimental Psychology, 22, 167-176.

Murdock, B. B, JR, W WaLker, K. D. (1969). Modality effects in free recall. Joumal of Verbal Leaming \& Verbal Behavior, 8, 665-676.

NAIRNE, J. S. (1988). A framework for interpreting recency effects in immediate serial recall. Memory \& Cognition, 16, 343-352.

NAIRNE, J. S. (1990). A feature model of immediate memory, Memory \& Cognition, 18, 251-269.

PENNEY, C. G. (1989). Modality effects and the structure of short-term verbal memory. Memory \& Cognition, 17, 398-422.

Shand, M. A., KLima, E. S. (1981). Nonauditory suffix effects in congenitally deaf signers of American Sign Language. Journal of Experimental Psychology: Human Leaming \& Memory, 7, 464-474.

Surprenant, A. M., Crowder, R. G. (1991). Failure to obtain a visual recency effecr. Unpublished manuscript, Department of Psychology, University, New Haven, CT.

WatKons, M. J. (1974). Locus of the modality effect in free recall. Journal of Verbal Learning \& Vertal Behavior, 11, 644-648. 


\section{NOTES}

1. I would like to thank Marco W. Battacchi for providing me with a copy of the raw data from Experiment 1 of Battacchi et al. (1990).

2. It should be noted that Battacchi et al. (1990) reported two experiments in addition to the one described above. These experiments included spatially distributed visual presentation, but they did not include fixed presentation control conditions. Consequently, it is not possible to determine whether distributed presentation enhanced the recency effect in either experiment. The absence of control conditions is critical because some of the recency effects found with fixed presentation in the present series of experiments are larger than the recency effects reported by Battacchi et al. in their final two experiments. For this reason, this report focuses exclusively on Battacchi et al.'s Experiment 1.
3. Direct comparisons between recency effects in fixed and distributed presentation conditions were evaluated with directional $t$ tests throughout this report because evidence for replication was sought.

4. As is suggested by the third panel of Figure 1, there was a difference in level of recall between distributed and fixed presentation, collapsing across serial position and mouthing condition $[.60$ vs. .54 ; $\left.F(1,27)=43.92, M S_{\mathrm{e}}=0.018, p<.001\right]$. This apparent difference should be treated with caution, however, because the analysis was entirely post hoc. More importantly, an analysis of the other six experiments failed to turn up any evidence that fixed and distributed presentation differed in overall level of recall. Thus, the result was not replicated.

(Manuscript received August 22, 1991; revision accepted for publication January 8,1992 .)

\section{Support for Cognitive Science Research National Institute of Mental Health}

The National Institute of Mental Health (NIMH) aims to support a wide range of basic research in the cognitive sciences as part of its effort to expand knowledge of fundamental aspects of human behavior and adaptation.

NIMH encourages proposals for basic research in such areas as perception, memory, knowledge, thinking, learning, language, and performance. Methods may include (but are not limited to) human and animal experimentation, computer simulation, and formal/computational analysis. Investigators may have backgrounds in psychology, computer science, linguistics, philosophy, or any other cognitive science.

Funding mechanisms include grants for research projects (of various sizes and durations), pre- and postdoctoral fellowships, institutional training grants, career scientist awards, and conference grants.

Questions about NIMH's support for cognitive science research can be directed to Rodney Cocking or Howard Kurtzman, Basic Behavioral and Cognitive Sciences Research Branch, National Institute of Mental Health, Room 11C-16, 5600 Fishers Lane, Rockville, MD 20857 (e-mail, kwh@cu.nih.gov; phone, (301)4439400; FAX, (301) 443-4822.)

\section{Members of Underrepresented Groups: Reviewers for Journal Manuscripts Wanted}

On behalf of Memory \& Cognition and Psychonomic Society Publications, I invite you to contact me if you are interested in reviewing manuscripts for Memory \& Cognition. Please send a letter and a copy of your curriculum vita to me at the following address: Memory \& Cognition, Department of Psychology, Indiana University, Bloomington, Indiana 47405. The letter or the vita should contain your complete address (including an electronic mail address if one is available), telephone number, and area(s) of expertise. Our reviewers have published articles in peer-reviewed journals, a standard prerequisite for being selected as a reviewer.

Please note that reviewing manuscripts takes time and must be completed quickly. If you are asked to review a manuscript, you will be expected to provide a thorough and prompt review.

Margaret Jean Intons-Peterson Editor 